\title{
Influencing Mechanism of Apparent Space Dimensions on Interface Aesthetics and Apparent Usability
}

\author{
Tian Lei ${ }^{1}$, Yingbin $\mathrm{Zhou}^{2}$, Xiang $\mathrm{Li}^{3}$, and Xiaoli Chen ${ }^{4}$ \\ ${ }^{1}$ Dept. of Industrial Design, Huazhong University of Science and Technology, \\ 430074 Wuhan, P.R. China \\ ${ }^{2}$ School of Art and Design, Wuhan Institute of Technolgy, 430073 Wuhan, P.R. China \\ ${ }^{3}$ School of Art and Design, Wuhan University of Technology 430070 Wuhan, P.R. China \\ ${ }^{4}$ Faculty of Mechanical and Electronic Information, \\ China University of Geosciences 430074 Wuhan, P.R. China \\ andrew.tianlei@gmail.com
}

\begin{abstract}
Apparent usability (AU) and interface aesthetics are the two important factors in HCI, which are affected by the apparent space dimension (ASD). This paper, by making two experiments, explored the influencing mechanism of ASD on them. The results show that: 1) AU is made up of subjective feelings, operation, and cognition; 2) interface aesthetics is made up of impression beauty, material beauty, and hominine beauty; 3) participants' subjective feelings increase with the addition of ASD; 4) participants have the strongest operational ability in the apparent 2-dimensional space but the weaker in the other two; 5) participants' cognition for the interactive system decrease with the addition of ASD; 6) interface's impression beauty increases with the addition of ASD; 7) interface's material beauty and hominine beauty are both the best in the apparent 2-dimensional space, but not good enough in the other two.
\end{abstract}

Keywords: apparent space dimension, apparent usability, interface aesthetics.

\section{Introduction}

Apparent Usability (AU) is an ignored branch of the Usability, which are the users' feelings or sensations about whether products or an interactive system is easy to use before using it. Just like Usability, AU has effect on users' acceptance and evaluation of an interactive system.

Aesthetics is another important factor in HCI. Recent work has suggested that aesthetics plays a considerable role in the interactive system. When a user faces an interactive system, the formal elements and their semantics will stimulate him immediately and cause him to produce perceptions and emotions, such as the overall impression, degree of preference, and interaction attitude[1],[2]. Gait reported that more interesting interface could increase a user's arousal and sustain his effectiveness and interest in the interactive system [3]; Jordan, with his experiments, found that aesthetics was the important determinant of whether an experience was pleasurable or not. Norman, D.A. thought that aesthetic design could affect a user's preference more than the traditional design based on Usability and operation performance [4]. 
Aesthetics can improve the interactive system's AU. For instance, Kurosu and Kashimura took the ATM's interface layout as independent variable, and apparent usability, inherent usability, actual usability, and aesthetics as dependent variables, and then made an experiment. The result indicated that there was a considerably high correlation between apparent usability and visual image (interface aesthetics) [5], [6].Tractinsky improved this experiment and got the similar results [7]. However, some other research finds that the relationship between them is not so. For example, Angeli etc. [8]found that the metaphor-based interface was better than the menu-based one in the expressive aesthetic dimension and users preferred the interface having a great attraction in the expressive aesthetic dimension although its inherent usability and apparent usability were less than the menu-based interface's.

Is there a potential factor that could affect $\mathrm{AU}$ and interface aesthetics at the same time and make the positive or negative correlation between them under different conditions? Through the initial screening, the factor of apparent space dimension (ASD) was brought into our study. ASD is the understanding of the number of interface's space dimensions, which is caused by the four key design techniques, gradients, shadows, perspective and directions of the movement. This paper, by making two experiments, will research the structure of AU, the influence of ASD on AU, and the relationship among ASD, AU, and interface aesthetics.

\section{Experiment}

This experiment is to research AU's structure and the influence of ASD on AU.

\subsection{Variables}

According to the four critical design techniques, the independent variable, ASD, was divided into three levels and each level had four classical values (layout), as shown in Table 1 and Fig. 1.

Table 1. Parameters of ASD

\begin{tabular}{lllll}
\hline Level & Gradients & Shadows & Perspective & Directions of the movement \\
\hline Level 1 & $\times$ & $\times$ & $\times$ & $\mathrm{X}$ or Y \\
Level 2 & $\sqrt{ }$ & $\times$ & $\times$ & $\mathrm{X}$ and $\mathrm{Y}$ \\
Level 3 & $\sqrt{ }$ & $\sqrt{ }$ & $\sqrt{ }$ & $\mathrm{X}, \mathrm{Y}$ and $\mathrm{Z}$ \\
\hline
\end{tabular}

The dependent variable was AU. It was composed of several different facets and each was corresponding to a question. According to Hassenzahl's 7-facet questionnaire [9] and Peiwen Lin's 6-facet questionnaire [10], three facets of pleasure, innovation and interest were involved into this experiment. The integral questionnaire is seen in Table 2. 


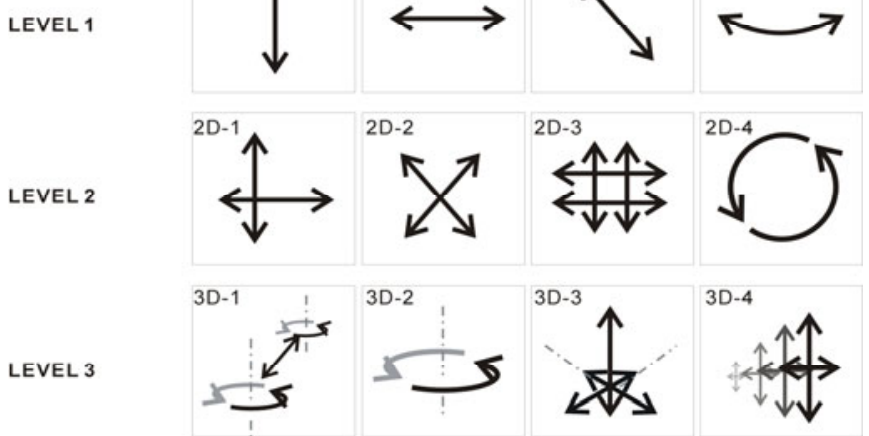

Fig. 1. Each value of the independent variable

There are many factors affecting the dependent variable. Here, icons' shapes, colors, texture, semantics, pix, and slide speed were the control variables which were controlled.

Table 2. The questionnaire of AU

\begin{tabular}{llll}
\hline No. & Questions & No. & Questions \\
\hline Q1 & Is it comprehensible? & Q7 & Is it familiar? \\
Q2 & Is it simple? & Q8 & Is it interesting? \\
Q3 & Is it ease- to-use? & Q9 & Does it give you the passion of usage? \\
Q4 & Is it clear? & Q10 & Is it creative? \\
Q5 & Is it safe? & Q11 Is it impressive after using it? \\
Q6 & Is it controllable? & Q12 & Does it give you the pleasant user experience? \\
\hline
\end{tabular}

\subsection{Materials and Participants}

Based on the above variables, 12 experimental samples were designed, as Fig. 2 shows. Each sample was corresponding to a value. Fifty-six college students (20-25 age), majoring in design, took part in this experiment.

\section{Results and Discussions of the Experiment}

\subsection{Structure of AU}

A PCA was conducted for the data from AU experiment (Table 3). Three main factors were extracted which could explain $38.996 \%, 28.209 \%$, and $24.547 \%$ of the total variance respectively after rotation. It suggests that participants' differences in AU caused by ASD can be totally explained $91.753 \%$ by these three main factors. The loadings of Q7, Q8, Q10, Q11, and Q12 on factor 1 were outstanding, which were $0.822,0.929,0.9,0.934$ and 0.819 respectively. These facets mainly reflect the 
participants' subjective understandings of interactive systems. So, it can be named subjective feelings factor (SFF). The loadings of Q1, Q3, Q6, and Q9 on factor 2 were bigger, which were $0.752,0.842,0.846$ and 0.853 respectively. These questions are closely correlated with users' operation behaviors, and accordingly factor 2 can be named operation factor $(\mathrm{OF})$. The contributions of Q2, Q4, and Q5 to factor 3 were bigger, and their factor loadings were $0.917,0.834$ and 0.927 in turn. These AU facets are closely related to users' cognitive loadings and information amount. So it can be called cognition factor (CF). Thus, we find that AU is mainly made up of three mutually independent components which are the subjective feelings factor, the operation factor and the cognition factor.
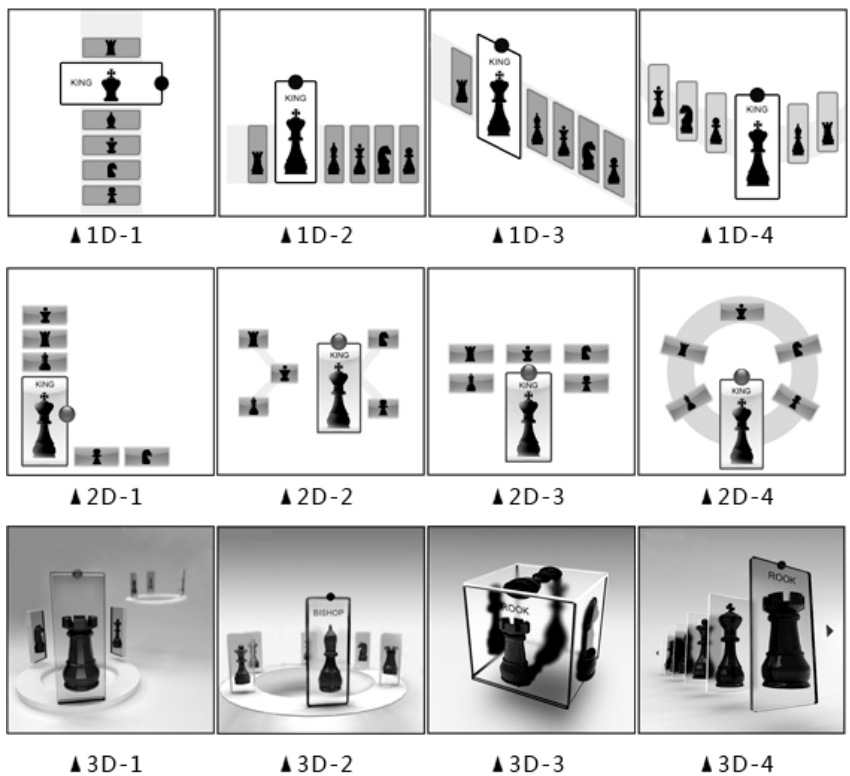

Fig. 2. Experimental samples

Table 3. Results of the Principal Component Analysis of AU facets

\begin{tabular}{cllllll}
\hline Component & \multicolumn{3}{c}{ Initial Eigenvalues } & \multicolumn{3}{c}{ Rotation Sums of Squared Loadings } \\
\cline { 2 - 7 } & Total & $\begin{array}{l}\text { \% of } \\
\text { Variance }\end{array}$ & $\begin{array}{l}\text { Cumulative } \\
\%\end{array}$ & Total & $\begin{array}{l}\% \text { of } \\
\text { Variance }\end{array}$ & $\begin{array}{l}\text { Cumulative } \\
\%\end{array}$ \\
\hline 1 & 6.922 & 57.682 & 57.682 & 4.680 & 38.996 & 38.996 \\
2 & 3.092 & 25.763 & 83.445 & 3.385 & 28.209 & 67.206 \\
3 & .997 & 8.309 & 91.753 & 2.946 & 24.547 & 91.753 \\
\hline
\end{tabular}

\subsection{Effect of ASD on AU}

What on earth does ASD influence AU? Taking the regressors of the AU's facets on three main factors as the new variables, seen in Table 4, and alternately choosing two 
factors from the three as coordinate axes, we got the samples' spatial distributions of AU, as shown in Fig.3, and Fig. 4.

Table 4. Regressors of the AU's facets on the three main factors

\begin{tabular}{llll}
\hline Independent variable & SFF & OF & CF \\
\hline 1D-1 & -.31457 & -1.05665 & 1.09496 \\
$1 D-2$ & .28325 & -.25887 & 1.67797 \\
$1 D-3$ & -1.62896 & -.68036 & .38898 \\
1D-4 & -.99274 & -1.12577 & .15391 \\
2D-1 & -.38316 & .63588 & -.44406 \\
2D-2 & -1.38397 & .27236 & -2.04399 \\
2D-3 & .19673 & 1.28346 & .89421 \\
2D-4 & -.11937 & 1.97979 & .17212 \\
3D-1 & 1.23741 & -.66683 & -.94712 \\
3D-2 & .61676 & .88486 & .06972 \\
3D-3 & 1.41528 & -.70276 & -.81447 \\
3D-4 & 1.07333 & -.56511 & -.20222 \\
\hline
\end{tabular}

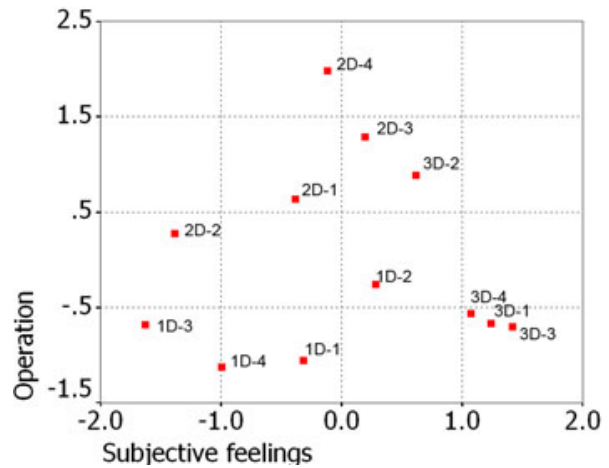

Fig. 3. The samples' distributions in SFF-OF space

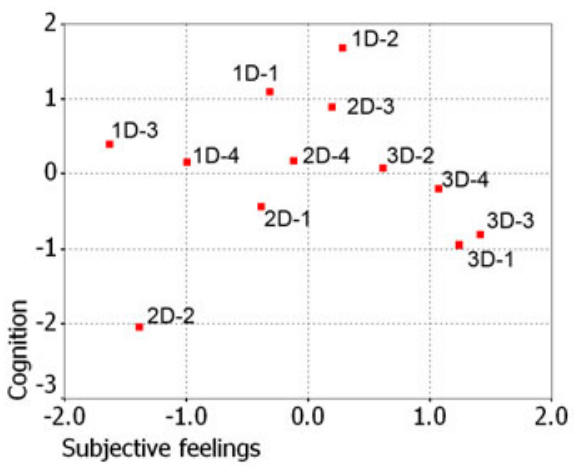

Fig. 4. The samples' distributions in SFF-CF space

Fig. 3 shows that: 1) Only the 2D-3 and 3D-2 two samples have the positive values on both SFF axis and OF axis, and only 1D-3, 1D-4, and 1D-1 are negative on the two axes; 2) the samples of 1D-2、3D-1、3D-3、3D-4 are all positive on SFF axis, and 3D-1, 3D-3, and 3D-4 are the top three on this axis, but their values on OF axis are all less than zero; 3 ) the samples of 2D-1、2D-2 and 2D-4 are located in the positive part of OF axis, and the value of sample 2D-4 is the highest in all the samples on this axis. Yet, they have a weaker performance on SFF axis.

Fig.4 shows that: 1) 1D-2、2D-3 and 3D-2 are all located in the positive part on both SFF axis and CF axis, and the cognition of sample 1D-2 is the best in all the samples. However, either the sample 2D-2 or the one 2D-1 has a negative value on the two axes, and the 2D-2 has the worst cognition; 2) 1D-1, 1D-3, 1D-4 and 2D-4 have a good performance on CF axis but a weak one on SFF axis; 3) The values of 3D-2, 3D-3 and 3D4 are all less than zero on $\mathrm{CF}$ axis, but greater than zero on SFF axis. 
Averaging each sample's new variables according to the number of ASD, we got Fig.5, Fig.6 and Fig.7. From it, we find that:

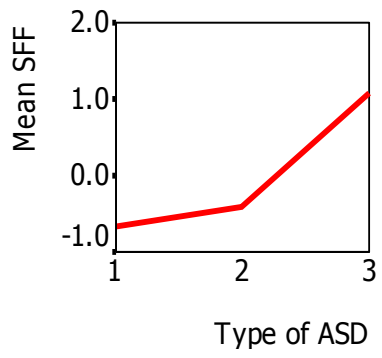

Fig. 5. The effect of ASD on SFF

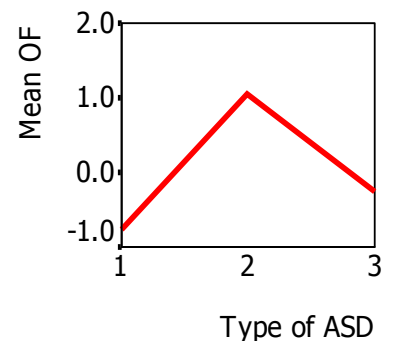

Type of ASD

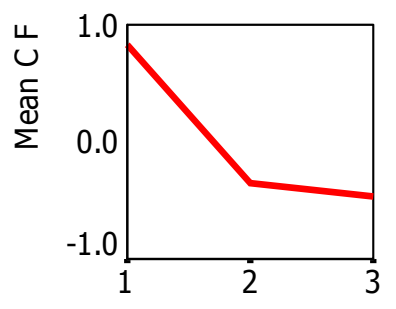

Type of ASD
Fig. 6. The effect of ASD on $\mathrm{OF}$
Fig. 7. The effect of ASD on $\mathrm{CF}$

1) The participants' subjective feelings caused by interface increase with the addition of the number of ASD.

2) The participants have the strongest confidence in controlling their operations in the apparent 2-dimensional space but the weaker in the apparent 1-dimensional space and the apparent 3-dimensional space.

3) The participants' understandings of the interactive system at the cognitive level decrease with the addition of the number of ASD

\section{Experiment}

The purpose of Experiment II is: 1) to verify the interface aesthetics' structure, and 2) to study the relationship between ASD and interface aesthetics. In order to find the influencing mechanism of ASD on both AU and interface aesthetics under the same conditions, we set up independent variables, control variables, experimental materials, and participants the same as Experiment I 's. Here, we used semantic differential to capture interface aesthetics.

30 descriptive aesthetic vocabularies (DAVs) were the dependent variables in this experiment, as shown in Table 5.

Table 5. The dependent variables of experiment

\begin{tabular}{|c|c|c|c|c|c|}
\hline No. & variable & No.variable & No. & variable & No. variable \\
\hline 1 & Harmonious & 9 Faddish & 17 & Steady & 25 Mysterious \\
\hline 2 & Unique & 10 Exalted & 18 & Dreamlike & 26 Classical \\
\hline 3 & Natural & 11 Smooth & 19 & Refreshing & 27 Decorative \\
\hline 4 & Elegant & 12 Exquisite & 20 & Fine & 28 Plain \\
\hline 5 & Avant-garde & 13 Feminine & 21 & Masculine & 29 Traditional \\
\hline 6 & Fresh and clean & 14 Magnificent & 22 & Stately & 30 Massive \\
\hline 7 & Concise & 15 Modern & 23 & Delicate and elegant & / \\
\hline 8 & Warm & 16 Rough & 24 & Lovely & \\
\hline
\end{tabular}




\section{Results and Discussions of the Experiment}

\subsection{Structure of Interface Aesthetics}

The previous study has shown that interface aesthetics is made up of impression beauty, material beauty and hominine beauty. It is confirmed by this experiment again. Data from Experiment II was dealt with PCA. Three main factors were extracted, and after rotation they respectively explained $46.005 \%, 19.78 \%$, and 18.07 $\%$ of the total variance produced by ASD, seen in table 6. Factor 1 was positively influenced by variables of "Unique", "Elegant", "Concise", "Faddish", "Exalted", "Smooth", "Feminine", "Magnificent", "Modern", "Dreamlike", "Fine”, "Lovely", "Mysterious", "Classical" and "Decorative" because the factor loadings of these DAVs on factor 1 were all greater than 0.7 . They were $0.953,0.789,0.889,0.982$, $0.952,0.814,0.906,0.866,0.952,0.862,0.871,0.914,0.873,0.853$, and 0.931 respectively. They convey a kind of formal beauty produced by association and empathizes. So, factor 1 is named impression beauty (IB). Factor 2 was affected by DAVs of "Harmonious", "Natural", "Fresh and clean", "Warm", "Exquisite", "Steady", and "Refreshing", whose factor loadings were 0.774, 0.909, 0.914, 0.811, 0.813, 0.685, and 0.611 respectively. These DAVs show a beauty caused by the physical situation and texture. So, factor 2 is called material beauty (MB). Factor 3 was influenced by "Avant-garde", "Rough", "Masculine", "Stately", "Plain", and "Traditional", and is named hominine beauty $(\mathrm{HB})$.

Table 6. Results of the PCA of experiment

\begin{tabular}{cllllll}
\hline $\begin{array}{l}\text { Compo- } \\
\text { nent }\end{array}$ & \multicolumn{2}{l}{ Initial Eigenvalues } & \multicolumn{3}{l}{ Rotation Sums of Squared Loadings } \\
\cline { 2 - 7 } & Total & $\begin{array}{c}\text { \% of } \\
\text { Variance }\end{array}$ & $\begin{array}{l}\text { Cumula- } \\
\text { tive \% }\end{array}$ & Total & $\begin{array}{l}\text { \% of } \\
\text { Variance }\end{array}$ & $\begin{array}{l}\text { Cumula- } \\
\text { tive \% }\end{array}$ \\
\hline 1 & 16.220 & 54.067 & 54.067 & 13.802 & 46.005 & 46.005 \\
2 & 6.903 & 23.010 & 77.076 & 5.934 & 19.780 & 65.785 \\
3 & 2.959 & 9.864 & 86.941 & 5.421 & 18.070 & 83.855 \\
\hline
\end{tabular}

\subsection{Effect of ASD on Interface Aesthetics}

What on earth does ASD affect interface aesthetics? Taking the regressors of the DAVs on the three main factors as new variables, seen in table 7, and alternately selecting two from the three as coordinate axes, we got the samples' spatial distributions of interface aesthetics, seen in Fig. 8, and Fig. 9.

Then averaging each sample's new variables according to the number of ASD, we find the clear relationship between ASD and interface aesthetics (Fig. 10, Fig. 11 and Fig. 12). As follows:

1) The impression beauty of interface increases as the number of ASD rises

2) The material beauty and the hominine one are both the best in the apparent 2dimensional space, and not good enough in neither the apparent 1-dimensional space nor the apparent 3-dimensional space. 
Table 7. Regressors of the DAVs on the three main factors

\begin{tabular}{llll}
\hline Independent variables & IB & MB & HB \\
\hline $1 \mathrm{D}-1$ & -.77819 & -1.37461 & -.54331 \\
$1 \mathrm{D}-2$ & -.54677 & .69628 & -1.78656 \\
$1 \mathrm{D}-3$ & -1.00528 & -1.33860 & .32201 \\
$1 \mathrm{D}-4$ & -1.16931 & .24226 & -1.24642 \\
$2 \mathrm{D}-1$ & -.93940 & .52454 & 1.06985 \\
$2 \mathrm{D}-2$ & -.98243 & .05102 & 1.46975 \\
$2 \mathrm{D}-3$ & .27215 & 1.37063 & 1.06031 \\
$2 \mathrm{D}-4$ & .41976 & 1.54666 & .18669 \\
$3 \mathrm{D}-1$ & 1.12506 & .14280 & -.75302 \\
$3 \mathrm{D}-2$ & 1.08128 & .20675 & -.65995 \\
$3 \mathrm{D}-3$ & 1.28975 & -1.14721 & .41238 \\
3D-4 & 1.23340 & -.92052 & .46827 \\
\hline
\end{tabular}
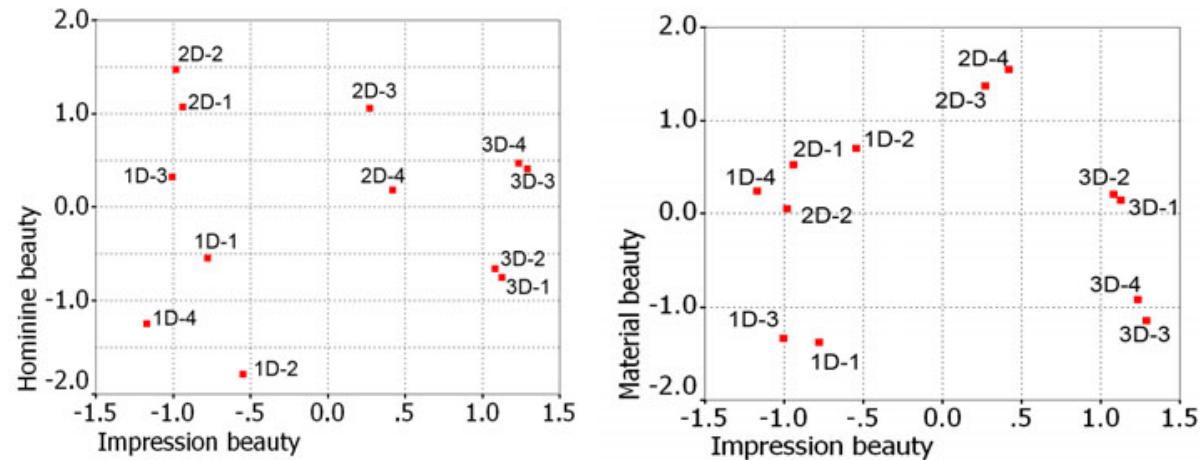

Fig. 8. The samples' distribution in IB-HB Fig. 9. The samples' distribution in IB-MB space space
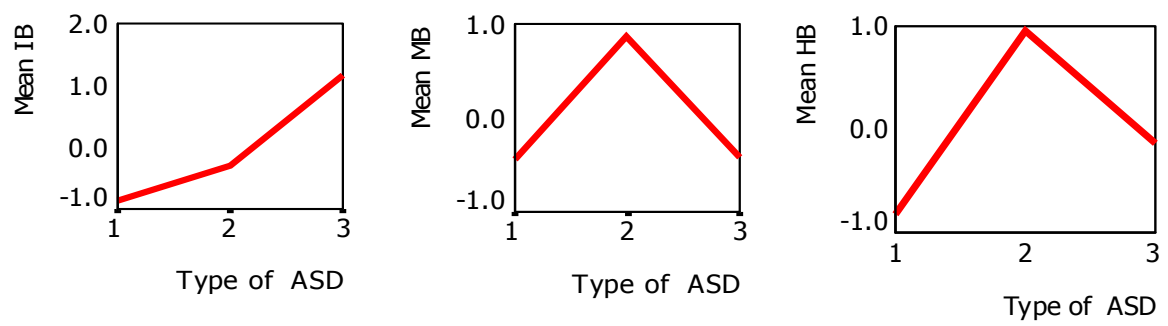

Fig. 10. The effect of ASD on Fig.11. The effect of ASD on Fig. 12. The effect of ASD on IB MB HB 


\section{Summary}

The above two experiments manifest that if changing the objects' gradients, shadows, directions of the movement, and perspective on interface, that is, increasing or decreasing the number of ASD, an interactive system's AU and aesthetics can make a different change accordingly. It seems that ASD determines the tones of AU and interface aesthetics.

How does interface aesthetics have effect on AU if the interactive system's ASD is decided? A Correlation Analysis was conducted among the six new variables, seen in Table 8. From it, we find that only between some specific factors does a statistically significant correlation occur. Impression beauty and subjective feelings are significant positive correlation at the 0.01 level (2-tailed), whose correlation coefficient $r$ is 0.893. Material beauty and operation factor of AU are also significant positive correlation at the 0.01 level (2-tailed), whose correlation coefficient $r$ is 0.772 .

Table 8. Results of the Correlation Analysis

\begin{tabular}{llllllll}
\hline & & IB & MB & HB & SFF & OF & CF \\
\hline IB & Pearson Correlation & 1 & .000 & .000 & $.893(* *)$ & .171 & -.172 \\
& Sig. (2-tailed) &. & 1.000 & 1.000 & .000 & .595 & .593 \\
MB & Pearson Correlation & .000 & 1 & .000 & .007 & $.772(* *)$ & .121 \\
& Sig. (2-tailed) & 1.000 &. & 1.000 & .983 & .003 & .707 \\
HB & Pearson Correlation & .000 & .000 & 1 & -.194 & .400 & -.538 \\
& Sig. (2-tailed) & 1.000 & 1.000 &. & .546 & .198 & .071 \\
SFF & Pearson Correlation & $.893(* *)$ & .007 & -.194 & 1 & .000 & .000 \\
& Sig. (2-tailed) & .000 & .983 & .546 &. & 1.000 & 1.000 \\
OF & Pearson Correlation & .171 & $.772(* *)$ & .400 & .000 & 1 & .000 \\
& Sig. (2-tailed) & .595 & .003 & .198 & 1.000 &. & 1.000 \\
CF & Pearson Correlation & -.172 & .121 & -.538 & .000 & .000 & 1 \\
& Sig. (2-tailed) & .593 & .707 & .071 & 1.000 & 1.000 &. \\
\hline
\end{tabular}

These determined correlations provide a theoretical basis to enhance the usability of an interactive system quickly and effectively. For example, by adjusting interface's texture and impression beauty, a system's usability can be improved.

\section{References}

1. Schenkman, B.N., Jonsson, F.U.: Aesthetics and Preferences of Web Pages. J. Behavior and Information Technology 19(5), 367-377 (2000)

2. Heijden, H.V.D.: Factors Influencing the Usage of Websites: the Case of A Generic Portal in the Netherlands. J. Information and Management 40, 541-549 (2003)

3. Gait, J.: An Aspect of Aesthetics in Human-Computer Communications: Pretty Windows. J. IEEE Transactions on Software Engineering 11(8), 714-717 (1985)

4. Norman, D.A.: Emotional Design: Why We Love (or Hate) Everyday Things. Basic Books, New York( (2004) 
5. Kurosu, M., Kashimura, K.: Apparent Usability vs. Inherent Usability: Experimental Analysis on the Determinants of the Apparent Usability. In: Conference on Human Factors and Computing Systems, pp. 292-293. ACM Press, New York (1995)

6. Kurosu, M., Kashimura, K.: Determinants of the Apparent Usability. In: Proceedings of IEEE SMC, Vancouver, Canada, pp. 1509-1514 (1995)

7. Tractinsky, N.: Aesthetics and Apparent Usability: Empirically Assessing Cultural and Methodological Issues. In: ACM CHI Conference Proceedings on Human Factors in Computing Systems, pp. 115-122. ACM Press, New York (1997)

8. Angeli, A.D., Sutcliffe, A., Hartmann, J.: Interaction, Usability and Aesthetics: What Influences Users' Preferences? In: 6th ACM Conference on Designing Interactive Systems, pp. 271-280. ACM Press, New York (2006)

9. Hassenzahl, M., Platz, A., Burmester, M., Lehner, K.: Hedonic and Ergonomic Quality Aspects Determine Software's Appeal. In: CHI 2000 Conference Proceedings, pp. 201208. ACM Press, New York (2000)

10. Lin, P.W.: The Study of E-commerce Website Interface Design-Aesthetics vs. Usability, http://nctur.lib.nctu.edu.tw/handle/987654321/9860 